1 Transposon-induced inversions activate gene expression in Maize pericarp

$$
\text { Sharu Paul Sharma*, Tao Zuo* and Thomas Peterson }{ }^{* \dagger}
$$

$3 *$ Department of Genetics, Development and Cell Biology, Iowa State University, Ames, IA

$4 \quad 50011$

$5 \quad$ Department of Agronomy, Iowa State University, Ames, IA 50011

6

7 Abstract

8 Chromosomal inversions can have considerable biological and agronomic impacts including

9 disrupted gene function, change in gene expression and inhibited recombination. Here we

10 describe the molecular structure and functional impact of six inversions caused by Alternative

11 Transpositions between $p 1$ and $p 2$ genes responsible for floral pigmentation in maize. In maize

12 line $p 1-w w B 54$, the $p 2$ gene is expressed in anther and silk but not in pericarp, making the

13 kernels white. We identified inversions in this region caused by transposition of Ac and fractured

$14 A c(f A c)$ transposable elements. These inversions change the position of a $p 1$ enhancer and

15 activate the expression of $p 2$ in the kernel pericarp, resulting in red kernel color. We hypothesize

16 that these inversions place the $p 2$ gene promoter near a $p 1$ gene enhancer, thereby activating $p 2$

17 expression in kernel pericarp.

18 


\section{Introduction}

25 Transposable elements are segments of DNA that can move within a genome. The maize

26 Activator $(A c)$ and Dissociation $(D s)$ transposable elements are members of the $h A T$ transposon

27 super-family, which is widespread in eukaryotes (Rubin et al. 2001). Barbara McClintock

28 discovered these transposons while studying the phenomenon of chromosome breakage. She

29 identified $D s$ as a locus on the short arm of chromosome 9 in some maize stocks where

30 chromosome breaks occurred frequently. She also showed that $D s$ is dependent on another

31 element $A c$ which is autonomous and can itself transpose. The $A c / D s$ system was also reported to

32 induce a variety of chromosomal rearrangements, such as translocations, deletions, duplications,

33 and inversions (McClintock 1950, 1951). The autonomous Ac element is 4565 bp in length and

34 carries a complete transposase gene. Ds elements vary in size and internal sequence and lack a

35 functional transposase gene, making them non-autonomous (Lazarow et al. 2013). The Ac

36 transposase is known to bind to subterminal motif sequences of $A c / D s$ elements and then cut at

37 the transposon 5' and 3' TIRs (Terminal Inverted Repeats; 11 bp imperfect repeats) (Becker and

38 Kunze 1997). Ac transposase can recognize and act on the termini of a single element (Standard

39 Transposition), or the termini of two different elements (Alternative Transposition); for example,

40 the 5' end of $A c$ and the 3' end of a second nearby element such as Ds or fractured Ac (fAc) (Su

41 et al. 2018). Standard Transposition events change only the position of a single element, while

42 Alternative Transposition events can produce a variety of genome rearrangements, depending on

43 the relative orientations of the TE termini and the location of the target site. When two

44 transposons are in direct orientation, the internal-facing termini are present in a reversed

45 orientation compared to the termini of a single transposon. In this configuration, the two facing

46 termini can undergo Reversed-Ends Transposition (RET) (Huang and Dooner 2008; Zhang and 
47 Peterson 2004; Zhang et al. 2009) to induce deletions (Zhang, J. and Peterson 2005; Zhang, J. et

48 al. 2006), duplications (Zhang et al. 2013), Composite Insertions (Zhang et al. 2014; Su et al.

49 2018, 2020), inversions (Zhang and Peterson 2004; Yu et al. 2011) and reciprocal translocations

50 (Pulletikurti et al. 2009; Zhang et al. 2009). For example, Zhang et al. 2009 described 17

51 reciprocal translocations and two large pericentric inversions derived by RET from a progenitor

52 allele containing $A c$ and $f A c$ insertions in the maize $p l$ gene. The frequent occurrence of these

53 structural changes and the fact that $A c$ inserts preferentially in or near genic regions (Kolkman et

54 al. 2005) suggest that Alternative Transposition events may have a significant impact on the

55 genome and transcriptome. Additionally, inversions provide an opportunity to analyze the

56 function of cis-regulatory elements, such as enhancers, in a native (non-transgenic) context.

58 The maize $p 1$ and $p 2$ genes are closely linked paralogous genes located on the short arm of

59 chromosome 1 that originated by duplication of an ancestral $P^{\text {pre }}$ gene, approximately 2.75 mya

60 (Zhang, P. et al. 2000). These genes are separated by a $\sim 70 \mathrm{~kb}$ intergenic region and coincide

61 with a major QTL for levels of silk maysin, a flavone glycoside with antibiotic activity toward

62 corn earworm (Zhang et al. 2003; Meyer et al. 2007). Both $p 1$ and p2 encode highly similar

63 R2R3 Myb transcription factors involved in controlling the structural genes $c 2$, chi, and $a 1$,

64 encoding chalcone synthase, chalcone isomerase, and dihydro-flavonol reductase, respectively

65 (Dooner et al. 1991; Grotewold et al. 1994). These enzymes of the flavonoid biosynthetic

66 pathway produce red phlobaphene pigments in maize floral organs. $p l$ is expressed in maize

67 kernel pericarp, cob, and silk, while $p 2$ is active in anther and silk (Zhang, P. et al. 2000; Goettel

68 and Messing 2009). Different $p 1$ alleles are indicated by a two-letter suffix indicating their 
69 expression in kernel pericarp and cob glumes; for example, $p 1-w w$ specifies white (colorless)

70 pericarp and white cob, while $P 1-w r$ indicates white pericarp and red cob.

72 The robust visual phenotypes and abundance of alleles with Ac insertions (Athma et al. 1992;

73 Moreno et al. 1992) make the $p 1 / p 2$ cluster an ideal genetic system to analyze the genetic impact

74 of Alternative Transposition events. The $p 1-w w B 54$ allele has a deletion of $p 1$ exons 1 and 2

75 along with insertions of $A c$ and fractured $A c(f A c)$ elements upstream of $p 1$ exon 3 (Yu et al.

76 2011). Because exons 1 and 2 encode most of the essential Myb DNA binding domain

77 (Grotewold et al. 1991) their deletion renders the $p 1$ gene non-functional leading to white kernel

78 pericarp and white cob. The 5' $A c$ and 3' $f A c$ termini are in a reversed orientation, separated by a

79331 bp inter-transposon segment. These elements exhibit frequent RET, leading to chromosome

80 breakage and rearrangements such as deletions and inversions (Yu et al., 2011). Here, we used

81 the $p 1-w w B 54$ allele as a starting point to isolate a variety of $p 1 / p 2$ gain of function alleles.

82 Among these, we identified independent cases of inversions with varying degrees of red kernel

83 pigmentation, possibly due to the activation of $p 2$ in pericarp tissue. Here we describe the

84 detailed structures and $p 2$ expression characteristics of six inversion cases.

86 Materials and Methods

87 Screening for Inversions derived from RET

88 The inversion alleles described here were derived from $p 1-w w B 54$ (Figure 1). Stock J ( $p 1$ -

$89 w w[4 C o 63]$ r1-m3::Ds) (described in Zhang et al. 2003) was used as common genetic

90 background and to detect the presence of $A c$ by excision of $D s$ from $r 1-m 3$. The occurrence of

91 red kernel pericarp in $p 1-w w B 54$ was used as a visual screen for $p 2$ activation in the pericarp (see 
bioRxiv preprint doi: https://doi.org/10.1101/2021.03.08.434479; this version posted March 9, 2021. The copyright holder for this preprint (which was not certified by peer review) is the author/funder. All rights reserved. No reuse allowed without permission.

Materials and Methods in Su et al. 2020). p1-wwB54 has white kernels, but approximately 1 in 8 ears were found to have a single red kernel, and $\sim 1$ in 40 ears had a multi-kernel red sector

94 (Figure 1, F86). The occurrence of a sector of red-colored pericarp on single or multiple kernels

95 reflects the stage of ear and kernel development at which an activating mutation (e.g.

97 be inherited (Emerson 1917). The red kernels were selected and planted, and in cases where the

98 new structure was transmitted through meiosis, the resulting plants would produce whole ears

99 with red kernels (Figure 1, S25). The pericarp is maternal tissue and hence the red color

100 phenotype is independent of the pollination parent.

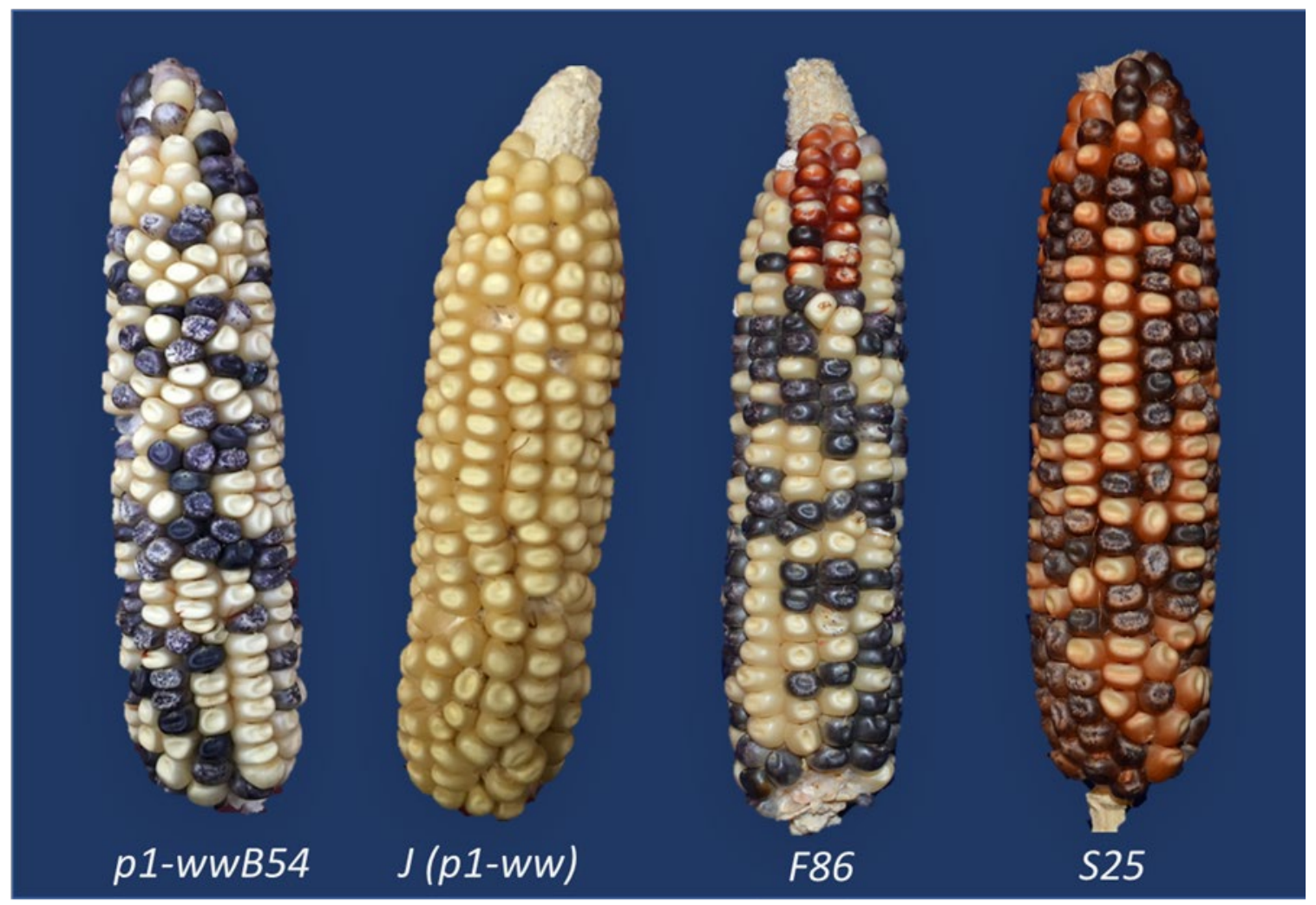

102 Figure 1: Ears of Different Maize $p 1 / p 2$ Alleles. Alleles $p 1$-wwB54 and $J(p 1-w w)$ have white (colorless)

103 kernel pericarp. F86 is a $p 1-w w B 54$ ear in which a sector of kernels near the ear tip has red pericarp due

104 to activation of $p 2$. S25 is an inversion allele with red pericarp color on the whole ear. Kernels with

105 purple-sectored aleurone are due to Ac-induced excision of Ds from r1-m3::Ds. 


\section{DNA extraction, Gel Electrophoresis, and Southern Blotting}

108 Genomic DNA was extracted from maize seedling leaves by a modified CTAB method (Saghai-

109 Maroof et al. 1984) and digested with different restriction enzymes according to the

110 manufacturer's instructions. For Southern blotting, genomic DNA digests were done with KpnI,

$111 H p a \mathrm{I}$, and EcoRV. Agarose gels (0.7\%) were run under 30 to 50 volts for 18 to 24 hours for

112 maximum separation of large fragments. The DNA was then transferred to a membrane for 24

113 hours, followed by probing each membrane with Fragment-15 (f15), a 411 bp sequence two

114 copies of which are located within the enhancer of the $p 1$ gene (Zhang F. and Peterson 2005).

$116 \quad P C R$, iPCR, and Sequencing

117 PCR was performed with $20 \mu \mathrm{L}$ reaction volumes under the following temperature conditions:

$1189^{\circ}$ for $2 \mathrm{~min}$, then $35 \mathrm{cycles}$ at $95^{\circ}$ for $30 \mathrm{sec}, 60^{\circ}$ for $30 \mathrm{sec}$, and $72^{\circ}$ for 1 min per $1-\mathrm{kb}$ length

119 of the expected PCR product, then final extension at $72^{\circ}$ for $5 \mathrm{~min}$. For initial PCR screening of

120 new alleles, a high-efficiency agarose gel electrophoresis method was used to visualize PCR

121 products (Sharma and Peterson 2020). Inversion breakpoint junctions ending with $f A c$ elements

122 were obtained by inverse-PCR (iPCR; Ochman et al. 1988). Inversion breakpoints at Ac

123 elements were isolated by Ac casting (Singh et al. 2003; Wang and Peterson 2013). This method

124 relies on the occurrence of frequent $A c$ transpositions to closely linked sites during plant

125 development. For each inversion, genomic DNA was isolated from seedling leaf tissue and then

126 the region containing the breakpoint was amplified by two pairs of nested PCR primers (Set 1

127 and then Nested in Table S1). The inversion breakpoint regions from I-PCR and Ac casting were 
sequenced by the Iowa State University DNA Sequencing Facility. Sequences were analyzed using Snapgene (snapgene.com) and BLAST (Zhang, Z. et al. 2000).

\section{RT-PCR Detection of 2 Expression}

132 Pericarps were peeled from kernels 15 to 20 days after pollination (DAP) and flash-frozen in

133 liquid nitrogen. Three biological replicates (pericarps from 3 sibling ears) were pooled to extract

134 RNA. RNA was isolated using Purelink Plant RNA Reagent, treated with NEB DNaseI, and

135 reverse transcribed to cDNA using Invitrogen ${ }^{\mathrm{TM}}$ SuperScript ${ }^{\mathrm{TM}}$ II Reverse Transcriptase kit using

136 protocols recommended by the product suppliers. Two technical replicates of reverse

137 transcription were used per sample. cDNAs were amplified by PCR using primers specific to

138 exons 1 and 3 of the $p 2$ gene transcript (Table S3). Primers specific to the maize Beta-tubulin

139 gene were used as an internal control.

\section{Data availability}

142 Maize genetic stocks are available by request to T.P. Sequences reported here are available in the

143 Supplemental Material.

\section{Results}

146 Due to the deletion of $p 1$ exons 1 and 2, the $p 1-w w B 54$ was expected to be a stable null allele.

147 We were surprised to see ears carrying $p 1-w w B 54$ produced red kernel pericarp sectors of

148 varying sizes (Figure 1). We hypothesized that the $p 2$ gene, which is normally not expressed in

149 kernel pericarp, could be activated by inversions generated by Reversed Ends Transposition

150 (RET) (Zhang and Peterson 2004; Zhang, J. and Peterson 2005; Zhang et al. 2009, 2013; Huang 
and Dooner 2008; Yu et al. 2011; Su et al. 2020). A diagram of this model showing an inversion

152 with breakpoints in the $p 2$ promoter region is shown in Figure 2. According to this model, RET

153 would begin with excision of the $A c 5^{\prime}$ end and $f A c 3^{\prime}$ end in $p 1-w w B 54$, followed by insertion of

154 the excised termini into a new target site unique for each event (Figure 2,a/b). If the 5' end of $A c$

155 (solid red arrowhead, Figure 2) joined with the ' $a$ ' side of the target sequence, and 3' end of $f A c$

156 (white arrowhead, Figure 2) joined with the ' $b$ ' side of the target site, the segment from 5' end of

$157 A c$ up to the target site $a / b$ will be inverted (for animation, see Supplemental data). The resulting

158 structure (Figure 2, Lower) contains an inversion of the $p 1-p 2$ interval; if the $p 2$ gene promoter

159 region is inserted sufficiently near the $p 1$ 3' pericarp enhancer (Sidorenko et al., 2000), p2 may

160 be expressed in the kernel pericarp.
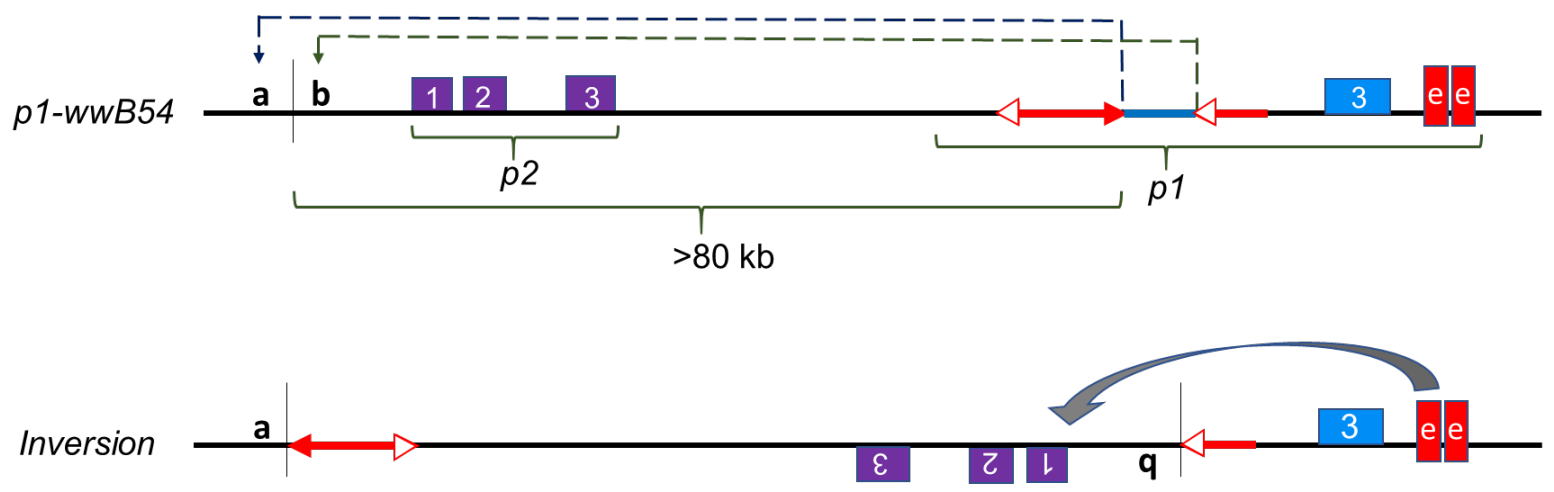

164 Figure 2: Model of RET-induced inversion leading to $p 2$ activation.

165 Upper: Diagram of progenitor allele p1-wwB54 and nearby p2 gene: Purple and blue boxes indicate 166 exons of $p 2$ and $p 1$ genes, respectively. Red arrows represent $A c$ (with two arrowheads) and fAc (with 167 single arrowhead) elements. Red boxes indicate two copies of $p 1$ enhancer fragment $f 15$. Dashed lines 168 indicate $A c / f A c$ excision by RET and re-insertion at $a / b$ target site upstream of $p 2$. The 331 bp DNA 
fragment between $A c$ and $f A c$ (blue line) is lost during the transposition event. The same symbols and

170 coloring scheme are used in other figures in this paper.

171 Lower: Inversion: Inverted segment extends from point a (Ac junction) to point $b$ (fAc junction) and

172 includes $A c, p 1-p 2$ intergenic region, and $p 2$ gene. In the inversion allele, the proximity of the $p 2$ promoter

173 to the $p 13^{\prime}$ enhancer may activate $p 2$ expression in the pericarp.

\section{Screening for Inversions}

176 To obtain RET-induced inversions, ears from several thousand plants carrying the $p 1-w w B 54$

177 allele were screened for kernels with red pericarp (example in Figure 1, third ear from left).

178 Selected red kernels were grown and propagated to obtain stable lines with various shades of red

179 kernel pericarp. Genomic DNA preparations from these lines were tested for structural

180 rearrangements by PCR using sets of primer pairs (Table S1) that can amplify the $A c$ and $f A c$

181 junctions in $p 1-w w B 54$ : primer set 1 detects the $p 1 / 3^{\prime} A c$ junction, and primer set 2 detects the

$1825^{\prime} A c / p 1 / 3^{\prime} f A c$ segment (Figure 3A). Simple $A c$ transposition or RET-induced deletion (Yu et

183 al., 2011) would result in negative PCR for both sets 1 and 2; while the formation of Composite

184 Insertions (Su et al., 2020) results in retention of both junctions. Whereas, RET-induced

185 inversion would result in retention of the $p 1 / 3^{\prime} A c$ junction (Set 1 positive), and loss of the 5'

$186 A c / p 1 / 3^{\prime} f A c$ segment (Set 2 negative). Using this test, several cases of putative inversions were

187 detected (Figure 3B). These cases were further tested using primers flanking the downstream

$188 f A c / p 1$ junction (Table S1) to confirm the retention of $f A c$ at its original position next to $p 1$ exon

189 3. Following confirmation of potential inversions, the new $A c$ and $f A c$ inversion breakpoint

190 junction sequences $(a / A c$ and $f A c / b$ in Figure $3 A)$ were amplified from genomic DNA using

191 direct PCR, Ac Casting, or iPCR (see Methods) along with nested PCR. Once obtained, both

192 inversion breakpoint junctions were sequenced (list of primers in Table S2). Junction sequences 
193 were examined to confirm expected orientations based on the established $p 1$ and $p 2$ genomic

194 sequence data (Zhang et al. 2006) and the presence of 8 bp TSDs (Target Site Duplication)

195 characteristic of $A c$ transposition (Figure 3A, yellow box and Table S4).

196

A

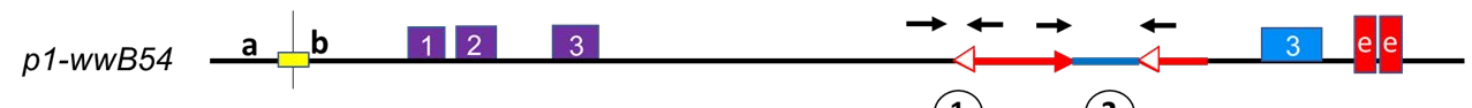

(1) 2

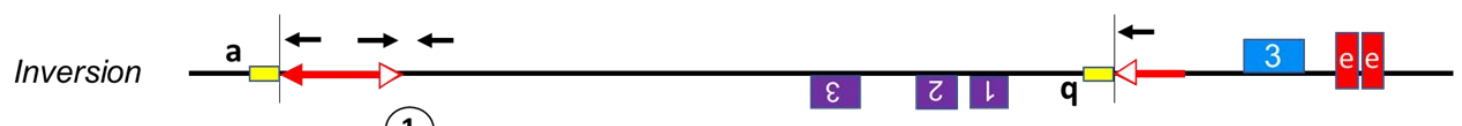

(1)

B $\quad 1 \quad 2 \quad 3 \quad 4 \quad 565$

(1)

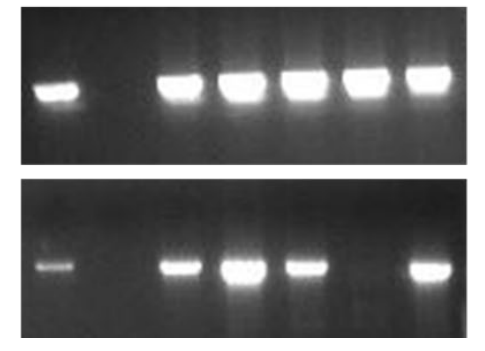

198 Figure 3: PCR Test for Inversions.

199 A) Progenitor $p 1$-wwB54 and derived Inversion allele structures showing locations of primers (black

200 arrows) used in PCR tests. Primer Set 1 detects the $p 1-A c$ junction which is present in both $p 1-w w B 54$

201 and Inversion; Primer Set 2 detects the Ac/p1/fAc segment which is present in $p 1$-wwB54 and absent in

202 Inversion. Yellow box is the 8 bp target site duplicated in inversion.

203 B) Agarose Gel image showing an example PCR using Primer Set 1 (upper) and Primer Set 2 (lower).

204 Lane 1, positive control (p1-wwB54); Lane 2, negative control ( 1 1-ww Stock J); Lanes 3- 7, candidates

205 tested. Only lane 6 (allele 132, not one of the cases described here) is positive for Set 1, negative for Set

2062 , as expected for inversions. 


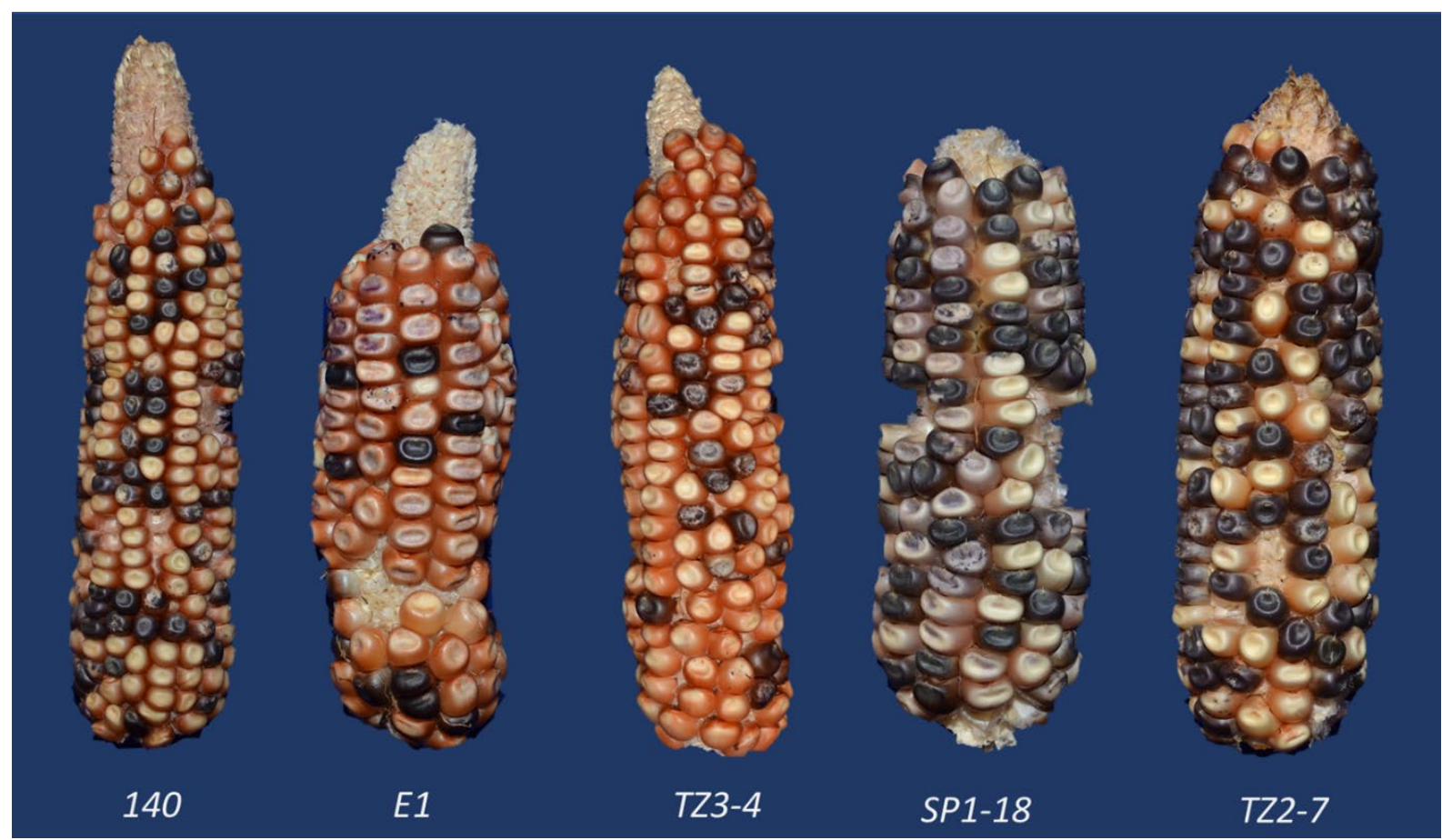

208 Figure 4: Representative Ears of Five Inversion Alleles. Ears have varying shades of red kernel pericarp

209 due to $p 2$ activation. The sixth inversion case S25 is shown in Figure 1. Some kernels have purple or

210 purple-sectored aleurone due to $A c$-induced excision of $D s$ from $r 1-m 3$ leading to anthocyanin

211 pigmentation.

\section{$213 \quad$ Structure of Inversions}

214 The structures of six independent inversions with red kernel pericarp were determined. Ears

215 produced by plants carrying these inversions are shown in Figures 1 and 4. The inversion

216 junctions were PCR-amplified and sequenced as described above, and their sequences compared

217 with established $p 1$ and $p 2$ genomic sequences to identify the breakpoint locations. One

218 breakpoint common to all cases is at the 5' end of $A c$ (Figure 5, vertical blue line), as expected

219 for inversions originating by RET of $A c$ and $f A c$ elements. The second breakpoint unique to each

220 allele is at the transposition target site, located in a $\sim 1 \mathrm{~kb}$ window from 2.6 to $3.5 \mathrm{~kb}$ upstream of

221 the $p 2$ transcription start site in these six cases (Figure 5, vertical red lines). These inversions 
222 reduce the distance between the $p 2$ transcription start site and the $p 1$ enhancer from $83.3 \mathrm{~kb}$ in

223 the parental $p 1-w w B 54$ allele to less than $10 \mathrm{~kb}$ in the inversion alleles (Figure 5 and Table S4).

224 The inverted fragment size ranges from 80.9 to $81.8 \mathrm{~kb}$. Each inversion allele contains an $8 \mathrm{bp}$

225 repeat sequence at the inversion junctions, precisely at the ends of the $A c$ and $f A c$ termini (Table

226 S4). These 8 bp repeats represent the signature Target Site Duplications (TSDs) resulting from

227 the staggered DNA cut made by $A c$ transposase. The presence of matching breakpoint TSDs

228 confirms that each inversion originated from a single Alternative Transposition event involving

229 the $A c / f A c$ elements.

230

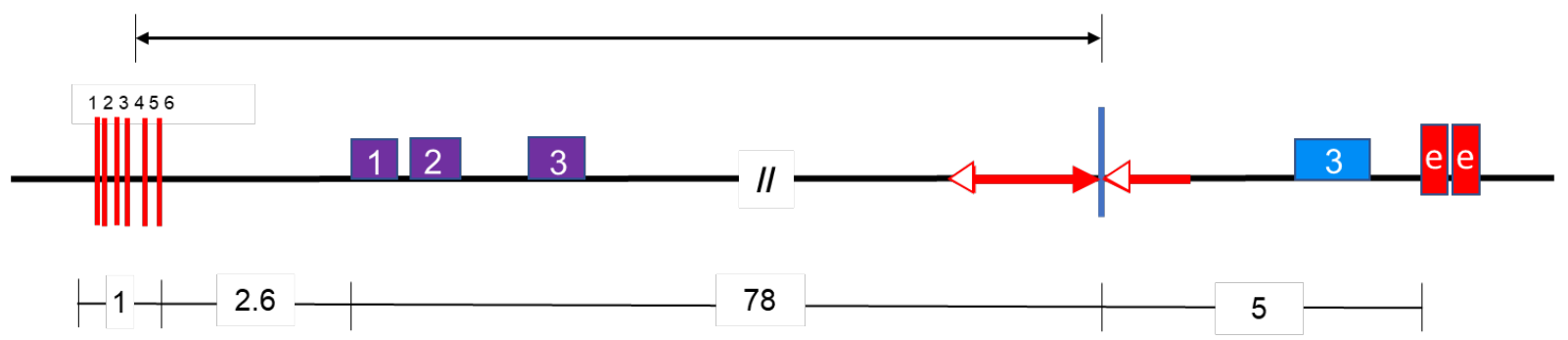

232 Figure 5: Map of the six inversion alleles. The vertical blue line is one breakpoint, and the red lines

233 indicate the second breakpoint unique to each inversion. Numbers on red lines correspond to alleles, 1 ,

234 140; 2, E1; 3, TZ3-4; 4, SP1-18; 5, S25; 6, TZ2-7. Numbers below the figure are distances in kbs.

236 After identifying the endpoints of the inversions, Southern blotting experiments were conducted

237 to examine the internal structures of the inverted fragments. Endonuclease $K p n I$ has recognition

238 sites located such that the unique inversion breakpoint and the $p l$ enhancer are contained in the

239 same restriction fragment in all six cases of inversions (Figure 6). This inversion junction

240 fragment was detected by hybridization with fragment-15 (f15) from within the $p 1$ enhancer. As

241 shown in Figure 6A, P1-rr4B2 (lane 3) gives two bands of size $6.3 \mathrm{~kb}$ and $8.6 \mathrm{~kb}$ as expected

242 because it has two copies of the enhancer, one on each side (5' and 3') of the $p 1$ gene (Figure 
6B; Sidorenko et al., 2000). Whereas the inversion progenitor $p 1-w w B 54$ (lane 4) gives a single

244 band of $13.5 \mathrm{~kb}$ representing the 3' enhancer fragment; the 5' enhancer fragment is deleted in

245 this allele (Figure 6C). The six inversion alleles (lanes 5 - 10) have progressively decreasing

246 band sizes, ranging from 12 to $10.5 \mathrm{~kb}$, reflecting the size differences resulting from different

247 junction breakpoints ' $b$ ' in each inversion (Figure 6D). Similar results were obtained using other

248 restriction enzymes including $H p a \mathrm{I}$ and $E c o$ RV (Figure S1) and probes $(A c-H$ for the $A c$

249 element, and $p 1$ Fragment $8 b$ for $p 2$ intron 2; not shown). All the results are consistent with the

250 presence of a simple inversion in each of these six cases, with no evidence of additional

251 rearrangements.

252

A

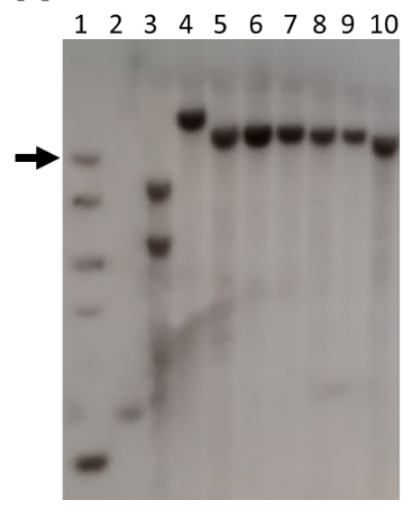

B

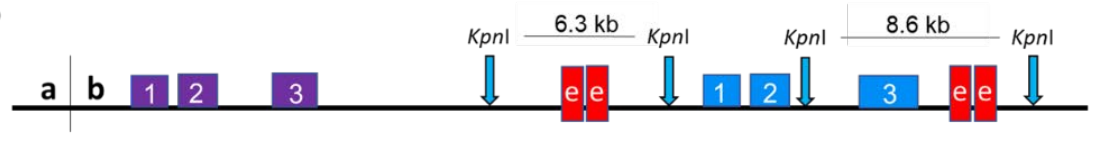

C

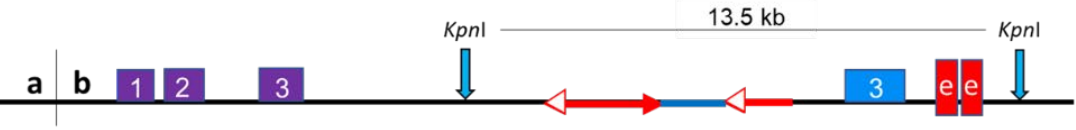

D

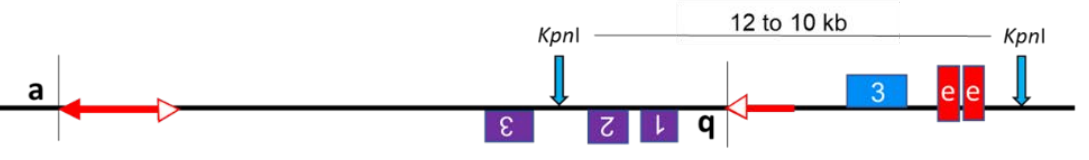

254 Figure 6: Genomic Southern Blot Analysis of Inversion Alleles.

255 A) Southern blot of genomic DNA samples from inversion homozygotes digested with Kpnl and probed

256 with fragment $f 15$ from the $p 1$ enhancer (red boxes in B, C and D). Lane 1, DNA ladder (arrow points to

$25710 \mathrm{~kb}$ band); Lane 2, J ( $p 1-w w)$; Lane 3, P1-rr4B2; Lane 4, progenitor $p 1-w w B 54$ (top band is $13.5 \mathrm{~kb}$ );

258 Lane 5, 140; Lane 6, E1; Lane 7, TZ3-4; Lane 8, SP1-18; Lane 9, S25; Lane 10, TZ2-7. The six

259 inversions (Lanes $5-10$ ) are arranged in order of decreasing band sizes (from 12 to $10.5 \mathrm{~kb}$ ).

260 B, C and D) Diagrams showing Kpnl restriction sites (vertical blue arrows) in B) P1-rr4B2, C) progenitor $261 \quad p 1-w w B 54$ and D) inversions. Southern blot band sizes reflect differences in the sites of $f A c$ insertion in 262 the $p 2$ promoter (breakpoint $b)$. 


\section{4 p2 Expression in Inversions}

265 The expression of the $p 2$ gene in plants homozygous for the inversion alleles was analyzed by

266 RT-PCR. RNA was extracted from pericarps of homozygous plants collected 15-20 DAP (days

267 after pollination) (Figure 7). P1-rr4B2 was used as a positive control (Figure 7, lane 2) because

$268 p 1$ is expressed in $P 1-r r 4 B 2$ pericarp and the same $p 2$ primers can amplify $p 1$ transcripts due to

269 sequence similarity. The six inversion alleles were derived from the $p 1-w w B 54$ maize line which

270 has a deleted $p 1$ gene and intact $p 2$ gene. The $p 2$ gene transcript was not detected in the pericarp

271 tissue of $p 1-w w B 54$ (Figure 7, lane 3), confirming previous results that $p 2$ is normally not

272 expressed in kernel pericarp (Zhang, P. et al. 2000). However, p2 transcripts were seen in all six

273 inversion cases (Figure 7, lanes 4 -9). To confirm the origin of these transcripts, the RT-PCR

274 product of one inversion was sequenced and found to have sequence polymorphisms matching

275 the $p 2$ gene (Figure S2). These results show that, unlike the progenitor $p 1-w w B 54, p 2$ is

276 expressed in the pericarp tissue of all six inversion alleles. This ectopic $p 2$ expression likely

277 resulted from the proximity of the $p 2$ gene promoter within the inverted fragment to the $p 13$ '

278 enhancer. In the progenitor $p 1-w w B 54$, the $p 2$ promoter region and $p 13$ ' enhancer are separated

279 by $83.3 \mathrm{~kb}$, whereas in the inversion alleles, this distance was reduced to between 7.4 and $8.2 \mathrm{~kb}$.

280 These results demonstrate the unique ability of inversions to modify gene expression near

281 inversion breakpoints by changing the distance from regulatory elements to their target genes. 


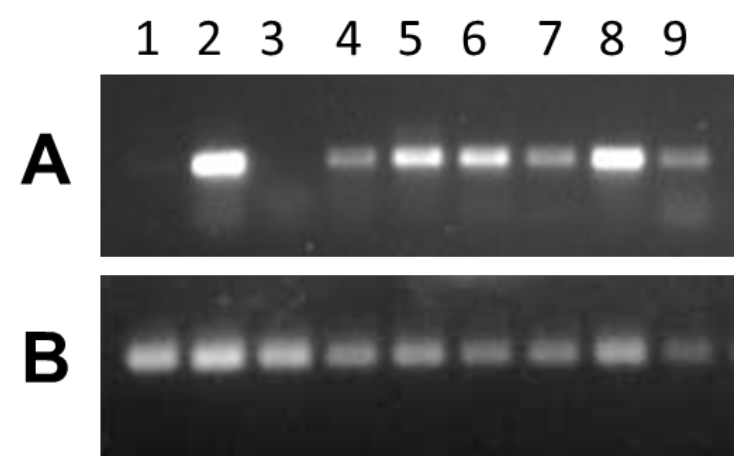

284 Figure 7: RT-PCR. Agarose gel images showing RT-PCR results using RNA extracted from pericarp

285 tissue and reverse transcribed to cDNA. PCR with primers from A) p2 exons 1 and 3, B) Beta-tubulin as

286 an internal control. Lane 1, J $(p 1-w w)$ is negative control; Lane 2, P1-rr4B2 is positive control for $p 1$

287 expression; Lane 3, p1-wwB54 is the progenitor and lacks p2 expression; Lane 4, 140; Lane 5, E1; Lane

288 6, TZ3-4; Lane 7, SP1-18; Lane 8, S25; and Lane 9, TZ2-7. All six inversion alleles are positive for $p 2$

289 expression.

291 Discussion

292 Mechanisms of inversions

293 A variety of molecular mechanisms are known to induce inversions. The double-strand break

294 (DSB) mechanism involves breakage and then repair by Non-Homologous End Joining (NHEJ)

295 (Moore and Haber 1996). If two double-strand breaks occur on the same chromosome, re-

296 ligation of the DNA molecule via NHEJ can form inversions (Hefferin and Tomkinson 2005),

297 deletions, or inversions flanked by inverted duplications, if the DSBs are staggered cuts (Ranz et

298 al. 2007). Additionally, inversions can result from ectopic recombination (Non-Allelic

299 Homologous Recombination, NAHR) between dispersed repeated sequences including

300 transposons (Delprat et al. 2009), retrotransposons (Kupiec and Petes 1988), interspersed repeat

301 sequences (Montgomery et al. 1991), or interspersed duplications (Cáceres et al. 2007). For

302 example, NAHR between pairs of homologous TEs present in opposite orientations at different 
303 positions on a chromosome can lead to inversions of the DNA segment between the two TEs

304 (Delprat et al., 2009). Recently, CRISPR has also been used to induce inversions in mammals

305 (Guo et al. 2015) and maize (Schwartz et al. 2020).

307 Here we show that DNA transposons, in addition to serving as passive substrates for ectopic

308 recombination, also directly induce inversions via Alternative Transposition reactions. Our

309 results are consistent with a model of RET-induced inversion, in which the ends of two nearby

310 DNA transposons are involved in a single transposition reaction. In this model, two TE copies

311 present in direct orientation will have their adjacent termini in a reversed orientation (i.e., the 5'

312 end of one TE faces 3' end of a second TE). Recognition of the terminal sequences of the two

313 TEs by the transposase will lead to an RET event in which the TE termini facing each other

314 attempt to transpose to a genomic target site. Because each TE remains linked to the donor

315 sequences by one un-transposed end, RET results in inversion of a flanking segment, and loss of

316 the fragment originally between the two TEs (Figure 2). Specifically, the DNA segment from

317 one TE end to the new insertion site is inverted. The resulting inversion has TEs present at each

318 breakpoint; one within the inversion and another just outside the second endpoint (Figure 6D).

319 The TE insertion is accompanied by a Target Site Duplications (TSD) flanking the TE termini at

320 the inversion breakpoints. As in standard transposition, the TSD is a result of the staggered cut

321 made by transposase followed by gap-filling and DNA ligation (Lazarow et al., 2013).

323 There are several important differences between inversions resulting from ectopic recombination

324 (NAHR) between two inversely oriented TEs and those caused by RET. First, inversions formed

325 by NAHR will not have a newly generated TSD; instead, the TSDs flanking the internal TE 
326 termini will also be inverted, resulting in TEs with (usually) non-identical TSDs. Second,

327 NAHR between two inversely oriented TEs can only flip the intervening segment; whereas, RET

328 can induce inversions of varying lengths on either side of each TE. Third, RET will only operate

329 on Class II TEs that transpose via "cut-and-paste" mechanism, and will not occur with Class I

330 elements that utilize a retro-transposition mechanism. Fourth, RET requires the expression of a

331 DNA transposase and transposition-competent TE termini in appropriate orientation; whereas,

332 NAHR proceeds via the action of host recombination machinery on substrate sequences of

333 sufficient homology and orientation.

334

335 The maize $A c / D s$ system is not the only known system that can cause inversions and other

336 rearrangements. Like $A c / D s$ elements in maize, the P-elements in Drosophila are also known to

337 cause inversions and other chromosomal rearrangements through Alternative Transpositions

338 (Gray et al. 1996; Tanaka et al. 1997). Other examples of such rearrangements via non-standard

339 transposition include impala elements in the fungus Fusarium (Hua-Van et al. 2002) and

340 Sleeping Beauty transposons in transgenes of mice (Geurts et al. 2006).

\section{1}

342 In addition to RET, the $A c / D s$ elements can also undergo Sister Chromatid Transposition (SCT)

343 (Zhang, J. and Peterson 2005; Zhang et al. 2013). While RET targets TEs on the same

344 chromosome, SCT involves TEs on sister chromatids. After DNA replication, a pair of Ac 5' and

345 3' termini in direct orientation can move to an un-replicated region where they can undergo a

346 second round of replication. This results in inverted duplications and Composite Insertions

347 (Wang et al. 2020). Both SCT and RET can lead to major rearrangements in the genome.

348 Transposition in the $A c / D s$ system is non-random (Vollbrecht et al. 2010) as Ac transposes 
349 preferentially into hypomethylated DNA (Kolkman et al. 2005) often associated with genic

350 regions (Cowperthwaite et al. 2002). This insertion preference likely increases the potential

351 genetic impact of $A c / D s$-induced Alternative Transposition events.

354 In a previous study, Yu et al. (2011) screened alleles with reverse-oriented $A c / f A c$ insertions in 355 an active $p 1$ gene for RET-induced loss of function mutants. Out of 100 mutants obtained, 89

356 were identified to have undergone major structural changes. Approximately half (47 out of 89)

357 were inversions, and the rest were primarily deletions plus some other rearrangements. This

358 result is consistent with the RET model which predicts that inversions and deletions are equally

359 likely to occur, because the outcome is determined by which transposon end $(A c$ or $f A c)$ is

360 ligated to which side ( $a$ or $b$ ) of the transposition target site. Here, we screened ears from

361 roughly 4000 plants of $p 1-w w B 54 / p 1-w w(J)$ genotype for red kernels indicating putative

362 rearrangements. About 400 unique red kernel events were found and propagated. The red

363 pericarp phenotype was inherited in 97 cases; 83 of these were characterized as rearrangements

364 due to RET. Among these 83, only 14\% (12 out of 83) were inversions, 35\% (29) were deletions,

365 and 51\% (42) were Composite Insertions. The markedly different proportion of inversions

366 recovered here (14\%) compared to Yu et al. $2011(53 \%)$ is most likely due to the different

367 screens used to detect RET events. The 2011 study began with a functional $p 1$ gene and selected

368 for loss-of-function events, yielding mostly deletions and inversions; most Composite Insertions

369 would not be detected because they leave the original donor locus intact (Zhang et al. 2014; Su et

370 al. 2018, 2020). Whereas, this study began with a non-functional $p 1$ allele, and required gain-of-

371 function (red pericarp sectors). This selection favored recovery of $p$ 2-expressing alleles caused 
372 by inversions and Composite Insertions near $p 2$ (Su et al., 2020). Indeed, all six of the cases

373 described here have inversion breakpoints within $3.5 \mathrm{~kb}$ upstream of the $p 2$ gene. This brings the

$374 p 2$ promoter to within $10 \mathrm{~kb}$ of the $p 1$ pericarp enhancer (Sidorenko et al., 2000), thus activating

375 the $p 2$ gene in a tissue in which it is not normally expressed.

377 The six inversion cases described here have no other detectable rearrangements. However, we 378 also obtained seven other cases of inversions which contain other more complicated structural 379 rearrangements. These cases of complex inversions are currently being characterized and will be 380 described elsewhere.

\section{Effects of Inversions on Fitness}

383 Inversions can have a variety of effects, such as causing position effect variegation of white gene

384 in Drosophila (Muller 1930; Levis et al. 1985; Lerach et al. 2006; Bao et al. 2007), suppressing

385 recombination (Jiang et al. 2007), and playing a vital role in the evolution of sex chromosomes

386 (Wright et al. 2016). Inversions are also associated with local adaptation and reproductive

387 isolation (Lowry and Willis 2010), as many closely related species are thought to have diverged

388 via inversion polymorphisms (Oneal et al. 2014; Twyford and Friedman 2015). Inversion of

389 boundary elements may also change higher-order organization in mammalian genomes, due to

390 the directional nature of CTCF binding sites (Guo et al. 2015). By altering topologically

391 associated domains (TAD) boundaries, inversions can cause misexpression and disease by

392 changing the relative position of enhancers and their target promoters (Lupiáñez et al. 2015;

393 Bompadre and Andrey 2019). 
395 Some inversions can result in major adaptive advantages; for example, the paracentric inversion

396 in Arabidopsis thaliana induced by Vandal transposon activity is strongly associated with

397 fecundity under drought conditions (Fransz et al. 2016). Inversions can even affect the spread of

398 disease: a chromosome 2La inversion in Anopheles gambiae is associated with susceptibility of

399 the vector to malaria infection (Riehle et al. 2017). Inversions are also involved in local

400 adaptation in teosinte populations (Pyhäjärvi et al. 2013). A large (13 Mb) inversion called

401 Inv4m found in Mexican highland maize populations affects expression of a large number of

402 genes regulating various developmental and physiological processes contributing to local

403 adaptation to highland environments (Crow et al. 2020).

404

405 The phlobaphene pigments controlled by the maize $p 1$ gene are non-essential, and many modern

406 corn varieties lack significant kernel pericarp color. However, a recent study reported that high

407 phlobaphene levels were associated with increased kernel pericarp thickness and reduced

408 mycotoxin contamination when compared to isogenic colorless pericarp lines lacking an active

409 pl gene (Landoni et al. 2020). Because the $p 1$ and $p 2$-encoded proteins are highly similar and

410 regulate the same flavonoid biosynthetic pathway (Zhang P. et al. 2000), similar effects are

411 likely induced by the expression of $p 2$ in the pericarp. Thus, the transposon-induced inversions

412 identified here may provide an adaptive benefit. Small $(<1 \mathrm{Mb})$ inversions are difficult to detect

413 by genetic and cytological methods, and so their frequency in plant populations is often

414 unknown. Our results show that even small, cytologically undetectable inversions between

415 linked genes may positively affect fitness. In summary, these findings suggest that Alternative

416 Transposition events may play a critical role in altering gene expression and generating adaptive

417 variation during genome evolution. 
419 Acknowledgments

420 We thank Jeremy Schuster and Matthew Johnston for field assistance, and Terry Olson for

421 technical assistance. This research is supported by the USDA National Institute of Food and

422 Agriculture Hatch project number IOW05282, and by State of Iowa funds.

423

\section{$424 \quad$ Literature Cited}

425 Athma, P., E. Grotewold, and T. Peterson, 1992 Insertional mutagenesis of the maize P gene by 426 intragenic transposition of Ac. Genetics, 131(1), pp. 199-209.

427

428 Bao, X., H. Deng, J. Johansen, J. Girton, K. M. Johansen, 2007 Loss-of-Function Alleles of the

429 JIL-1 Histone H3S10 Kinase Enhance Position-Effect Variegation at Pericentric Sites in

430 Drosophila Heterochromatin. Genetics, 176(2), pp. 1355-1358. doi:

$431 \quad 10.1534 /$ genetics.107.073676.

432

433 Becker, H. A. and R. Kunze, 1997 Maize Activator transposase has a bipartite DNA binding

434 domain that recognizes subterminal sequences and the terminal inverted repeats. Molecular

435 Genetics and Genomics, 254(3), pp. 219-230. doi: 10.1007/s004380050410.

436

437 Bompadre, O. and G. Andrey, 2019 Chromatin topology in development and disease. Current

438 Opinion in Genetics \& Development, Volume 55, pp. 32-38. doi: 10.1016/j.gde.2019.04.007. 
440 Cáceres, M., R. T. Sullivan, and J. W. Thomas, 2007 A recurrent inversion on the eutherian X

441 chromosome. Proceedings of the National Academy of Sciences of the United States of America,

442 104(47), pp. 18571-18576. doi: 10.1073/pnas.0706604104.

443

444 Cowperthwaite, M., W. Park, Z. Xu, X. Yan, S. C. Maurais et al., 2002 Use of the transposon Ac

445 as a gene-searching engine in the maize genome. Plant Cell, 14(3): 713-26. doi:

$446 \quad 10.1105 /$ tpc.010468.

447

448 Crow, T., J. Ta, S. Nojoomi, M. R. Aguilar-Rangel, J. V. T. Rodríguez et al., 2020 Gene

449 regulatory effects of a large chromosomal inversion in highland maize. PLOS Genetics, 16(12):

450 e1009213. https://doi.org/10.1371/journal.pgen.1009213

451

452 Delprat, A., B. Negre, M. Puig, and A. Ruiz, 2009 The transposon Galileo generates natural

453 chromosomal inversions in Drosophila by ectopic recombination. PLOS ONE, 4(11).

454 https://doi.org/10.1371/journal.pone.0007883.

455

456 Dooner, H. K., T. P. Robbins, and R. A. Jorgensen, 1991 Genetic and developmental control of

457 anthocyanin biosynthesis. Annual Review of Genetics, 25(1), pp. 173-199.

458 https://doi.org/10.1146/annurev.ge.25.120191.001133.

459

460 Emerson, R. A., 1917 Genetical studies of variegated pericarp in maize. Genetics 2: 1-35 
462 Fransz, P., G. Linc, C. R. Lee, S. A. Aflitos, J. R. Lasky et al., 2016 Molecular, genetic and 463 evolutionary analysis of a paracentric inversion in Arabidopsis thaliana. Plant Journal, 88(2), pp.

464 159-178. doi: 10.1111/tpj.13262.

465

466 Geurts, A. M., L. S. Collier, J. L. Geurts, L. L. Oseth, M. L. Bell et al., 2005 Gene Mutations and

467 Genomic Rearrangements in the Mouse as a Result of Transposon Mobilization from

468 Chromosomal Concatemers. PLOS Genetics, 2(9), pp. 1413-1423. doi:

469 10.1371/journal.pgen.0020156.

470

471 Goettel, W. and J. Messing, 2009 Change of gene structure and function by non-homologous

472 end-joining, homologous recombination, and transposition of DNA. PLOS Genetics, 5(6).

473 https://doi.org/10.1371/journal.pgen.1000516.

474

475 Gray, Y. H. M., M. M. Tanaka, and J. A. Sved, 1996 P-Element-Induced Recombination in

476 Drosophila melanogaster: Hybrid Element Insertion. Genetics, 144(4), pp. 1601-1610.

477

478 Grotewold, E., P. Athma, and T. Peterson, 1991 Alternatively spliced products of the maize P

479 gene encode proteins with homology to the DNA binding domain of Myb-like transcription

480 factors. Proc. Natl. Acad. Sci. 88: 4587-4591. https://doi.org/10.1073/pnas.88.11.4587.

481

482 Grotewold, E., B. J. Drummond, B. Bowen, and T. Peterson, 1994 The myb-homologous P gene

483 controls phlobaphene pigmentation in maize floral organs by directly activating a flavonoid

484 biosynthetic gene subset. Cell, 76(3), pp. 543-553. doi: 10.1016/0092-8674(94)90117-1. 
486 Guo, Y., Q. Xu, D. Canzio, J. Shou, J. Li et al., 2015 CRISPR Inversion of CTCF Sites Alters

487 Genome Topology and Enhancer/Promoter Function. Cell, 162(4), pp. 900-910. doi:

$488 \quad 10.1016 /$ j.cell.2015.07.038.

489

490 Hefferin, M. L. and A. E. Tomkinson, 2005 Mechanism of DNA double-strand break repair by

491 non-homologous end joining. DNA Repair, 4(6), pp. 639-648. doi:

492 10.1016/j.dnarep.2004.12.005.

493

494 Huang, J. T. and H. K. Dooner, 2008 Macrotransposition and Other Complex Chromosomal

495 Restructuring in Maize by Closely Linked Transposons in Direct Orientation. The Plant Cell, 496 20(8), pp. 2019-2032. doi: https://doi.org/10.1105/tpc. 108.060582

497

498 Hua-Van, A., T. Langin, and M. J. Daboussi, 2002 Aberrant transposition of a Tc1-mariner

499 element, impala, in the fungus Fusarium oxysporum. Molecular Genetics and Genomics, 267(1),

500 pp. 79-87. doi: 10.1007/s00438-002-0638-9.

501

502 Jiang, L., W. Zhang, Z. Xia, G. Jiang, Q. Qian et al., 2007 A paracentric inversion suppresses

503 genetic recombination at the FON3 locus with breakpoints corresponding to sequence gaps on

504 rice chromosome 11L. Molecular Genetics and Genomics, 277(3), pp. 263-272.

505 doi:10.1007/s00438-006-0196-7.

506 
507 Kolkman, J. M., L. J. Conrad, P. R. Farmer, K. Hardeman, K. R. Ahern et al., 2005 Distribution

508 of Activator (Ac) Throughout the Maize Genome for Use in Regional Mutagenesis. Genetics,

509 169(2), pp. 981-995. doi: 10.1534/genetics.104.033738.

510

511 Kupiec, M. and T. D. Petes, 1988 Allelic and ectopic recombination between Ty elements in

512 yeast. Genetics, 119(3), pp. 549-559.

513

514 Landoni, M., D. Puglisi, E. Cassani, G. Borlini, G. Brunoldi et al., 2020 Phlobaphenes modify

515 pericarp thickness in maize and accumulation of the fumonisin mycotoxins. Scientific Reports,

516 10(1), p. 1417. https://doi.org/10.1038/s41598-020-58341-8.

517

518 Lazarow, K., M. L. Doll, and R. Kunze, 2013 Molecular biology of maize Ac/Ds elements: an

519 overview. Methods of Molecular Biology, Volume 1057, pp. 59-82. doi: 10.1007/978-1-62703-

520 568-2_5.

521

522 Lerach, S., W. Zhang, X. Bao, H. Deng, J. Girton et al., 2006 Loss-of-function alleles of the JIL-

5231 kinase are strong suppressors of position effect variegation of the wm4 allele in Drosophila.

524 Genetics, 173(4), pp. 2403-2406. doi: 10.1534/genetics.106.059253

525

526 Levis, R., T. Hazelrigg, and G. Rubin, 1985 Effects of genomic position on the expression of

527 transduced copies of the white gene of Drosophila. Science, 229(4713), pp. 558-561. doi:

$528 \quad 10.1126 /$ science.2992080.

529 
530 Lowry, D. B. and J. H. Willis, 2010 A widespread chromosomal inversion polymorphism

531 contributes to a major life-history transition, local adaptation, and reproductive isolation. PLOS

532 Biology, 8(9), pp. 848-855. https://doi.org/10.1371/journal.pbio.1000500.

533

534 Lupiáñez, D. G., K. Kraft, V. Heinrich, P. Krawitz, F. Brancati et al., 2015 Disruptions of

535 Topological Chromatin Domains Cause Pathogenic Rewiring of Gene-Enhancer Interactions.

536 Cell, 161(5), pp. 1012-1025. doi: 10.1016/j.cell.2015.04.004.

537

538 McClintock, B., 1950 The origin and behavior of mutable loci in maize. Proceedings of the

539 National Academy of Sciences of the United States of America, 36(6), pp. 344-355.

540 https://doi.org/10.1073/pnas.36.6.344.

541

542 McClintock, B., 1951 Mutable Loci in Maize. Carnegie Inst. Wash. YearBook 49:157-167.

543

544 Meyer, J. D. F., M. E. Snook, K. E. Houchins, B. G. Rector, N. W. Widstrom et al., 2007

545 Quantitative trait loci for maysin synthesis in maize (Zea mays L.) lines selected for high silk

546 maysin content. Theor Appl Genet 115, 119-128. https://doi.org/10.1007/s00122-007-0548-7.

547

548 Montgomery, E. A., S. M. Huang, C. H. Langley, and B. H. Judd, 1991 Chromosome

549 rearrangement by ectopic recombination in Drosophila melanogaster: genome structure and

550 evolution. Genetics, 129(4), pp. 1085-1098. 
552 Moore, J. K. and J. E. Haber, 1996 Cell cycle and genetic requirements of two pathways of

553 nonhomologous end-joining repair of double-strand breaks in Saccharomyces cerevisiae.

554 Molecular and Cellular Biology, 16(5), pp. 2164-2173. doi: 10.1128/mcb.16.5.2164.

555

556 Moreno, M. A., J. Chen, I. Greenblatt, and S. L. Dellaporta, 1992 Reconstitutional mutagenesis

557 of the maize P gene by short-range Ac transpositions. Genetics, 131(4), pp. 939-956.

558

559 Muller, H. J., 1930 Types of visible variations induced by X-rays in Drosophila. Journal of

560 Genetics, 22, pp. 299-334. https://doi.org/10.1007/BF02984195.

561

562 Ochman, H., A. S. Gerber, and D. L. Hartl, 1988 Genetic applications of an inverse polymerase 563 chain reaction. Genetics, vol. 120 no. 3 621-623.

564

565 Oneal, E., D. B. Lowry, K. M. Wright, Z. Zhu, and J. H. Willis, 2014 Divergent population

566 structure and climate associations of a chromosomal inversion polymorphism across the

567 Mimulus guttatus species complex. Molecular Ecology, 23(11), pp. 2844-2860. doi:

$568 \quad 10.1111 / \mathrm{mec} .12778$.

569

570 Pulletikurti, V., C. Yu, J. Zhang, T. Peterson, and D. F. Weber, 2009 Cytological Evidence that

571 Alternative Transposition by Ac Elements Causes Reciprocal Translocations and Inversions in

572 Zea mays L. Maydica, 54(4), pp. 457-462.

573 
574 Pyhäjärvi, T., M. B. Hufford, S. Mezmouk, and J. Ross-Ibarra, 2013 Complex Patterns of Local

575 Adaptation in Teosinte. Genome Biol Evol.; 5(9): 1594-1609. doi: 10.1093/gbe/evt109

576

577 Ranz, J. M., D. Maurin, Y. S. Chan, M. v. Grotthuss, L. W. Hillier et al., 2007 Principles of

578 Genome Evolution in the Drosophila melanogaster Species Group. PLOS Biology, 5(6).

579 https://doi.org/10.1371/journal.pbio.0050152.

580

581 Riehle, M. M., T. Bukhari, A. Gneme, W. M. Guelbeogo, B. Coulibaly et al., 2017 The

582 Anopheles gambiae 2La chromosome inversion is associated with susceptibility to Plasmodium

583 falciparum in Africa. eLife, Volume 6. doi: 10.7554/eLife.25813.

584

585 Rubin, E., G. Lithwick, and A. A. Levy, 2001 Structure and Evolution of the hAT Transposon

586 Superfamily. Genetics, Volume 158, Issue 3, Pages 949-957.

587

588 Saghai-Maroof, M. A., K. M. Soliman, R. A. Jorgensen, and R. W. Allard, 1984 Ribosomal

589 DNA spacer-length polymorphisms in barley: mendelian inheritance, chromosomal location, and

590 population dynamics. Proceedings of the National Academy of Sciences of the United States of

591 America, 81(24), pp. 8014-8018. doi: 10.1073/pnas.81.24.8014.

592

593 Schwartz, C., B. Lenderts, L. Feigenbutz, P. Barone, V. Llaca et al. 2020 CRISPR-Cas9-

594 mediated 75.5-Mb inversion in maize. Nat. Plants 6, 1427-1431. doi:

595 https://doi.org/10.1038/s41477-020-00817-6. 
Sharma, S. P. and T. Peterson, 2020 Rapid Detection of Transposon-Induced Genome

598 Rearrangements. In press

599

600 Sidorenko, L. V., Li, X., Cocciolone, S. M., Tagliani, L., Chopra, S., Bowen, B., Daniels, M.,

601 and Peterson, T. 2000. Complex structure of a maize $M y b$ gene promoter: functional analysis in

602 transgenic plants. The Plant Journal 22: 471-482.

603

604 Singh, M., P. E. Lewis, K. Hardeman, L. Bai, J. K. C. Rose et al., 2003 Activator Mutagenesis of 605 the Pink scutellum1/viviparous7 Locus of Maize. The Plant Cell, 15(4), pp. 874-884. doi:

$606 \quad 10.1105 /$ tpc.010249.

607

608 Su, W., S. P. Sharma, and T. Peterson, 2018 Evolutionary Impacts of Alternative Transposition.

609 In: Pontarotti P. (eds) Origin and Evolution of Biodiversity. Springer, Cham.

610 https://doi.org/10.1007/978-3-319-95954-2_7.

611

612 Su, W., T. Zuo, and T. Peterson, 2020 Ectopic Expression of a Maize Gene Is Induced by

613 Composite Insertions Generated Through Alternative Transposition. Genetics, 216(4), pp. 1039-

614 1049. https://doi.org/10.1534/genetics.120.303592.

615

616 Tanaka, M. M., X. M. Liang, Y. H. M. Gray, and J. A. Sved, 1997 The accumulation of P-

617 element-induced recombinants in the germline of male Drosophila melanogaster. Genetics, 618 147(4), pp. 1769-1782. 
620 Twyford, A. D. and J. Friedman, 2015 Adaptive divergence in the monkey flower Mimulus

621 guttatus is maintained by a chromosomal inversion. Evolution, 69(6), pp. 1476-1486. doi:

$62210.1111 /$ evo.12663.

623

624 Vollbrecht, E., J. Duvick, J. P. Schares, K. R. Ahern, P. Deewatthanawong et al., 2010 Genome-

625 Wide Distribution of Transposed Dissociation Elements in Maize. The Plant Cell, 22(6), pp.

626 1667-1685. doi: https://doi.org/10.1105/tpc.109.073452.

627

628 Wang, D. and T. Peterson, 2013 Isolation of sequences flanking Ac insertion sites by Ac casting. 629 Methods of Molecular Biology, Volume 1057, pp. 117-122. doi: 10.1007/978-1-62703-568-2_8.

631 Wang, D., J. Zhang, T. Zuo, M. Zhao, D. Lisch and T. Peterson, 2020 Small RNA-Mediated De

632 Novo Silencing of Ac/Ds Transposons Is Initiated by Alternative Transposition in Maize

633 Genetics, 215 (2), pp. 393-406. doi: https://doi.org/10.1534/genetics.120.303264

634

635 Wright, A. E., R. Dean, F. Zimmer, and J. E. Mank, 2016 How to make a sex chromosome.

636 Nature Communications, 7, pp. 12087. https://doi.org/10.1038/ncomms12087.

637

638 Yu, C., J. Zhang, and T. Peterson, 2011 Genome Rearrangements in Maize Induced by

639 Alternative Transposition of Reversed Ac/Ds Termini. Genetics, 188(1), pp. 59-67. doi:

$640 \quad 10.1534 /$ genetics.111.126847.

641 
642 Zhang, F. and T. Peterson, 2005 Comparisons of Maize pericarp color1 Alleles Reveal

643 Paralogous Gene Recombination and an Organ-Specific Enhancer Region. The Plant Cell, 17 (3)

644 903-914; doi: 10.1105/tpc.104.029660.

645

646 Zhang, J. and T. Peterson, 2004 Transposition of reversed Ac element ends generates

647 chromosome rearrangements in maize. Genetics, 167(4), pp. 1929-1937. doi:

648 10.1534/genetics.103.026229.

649

650 Zhang, J. and T. Peterson, 2005 A Segmental Deletion Series Generated by Sister-Chromatid

651 Transposition of Ac Transposable Elements in Maize. Genetics, 171(1), pp. 333-344.

652 https://doi.org/10.1534/genetics.104.035576.

653

654 Zhang, J., F. Zhang, and T. Peterson, 2006 Transposition of Reversed Ac Element Ends

655 Generates Novel Chimeric Genes in Maize. PLOS Genetics, 2(10), pp. 1535-1540.

656 https://doi.org/10.1371/journal.pgen.0020164.

657

658 Zhang, J., C. Yu, V. Pulletikurti, J. Lamb, T. Danilova et al., 2009 Alternative Ac/Ds

659 transposition induces major chromosomal rearrangements in maize. Genes \& Development,

660 23(6), pp. 755-765. doi: 10.1101/gad.1776909

661

662 Zhang, J., T. Zuo, and T. Peterson, 2013 Generation of tandem direct duplications by reversed-

663 ends transposition of maize ac elements. PLOS Genetics, 9(8).

664 https://doi.org/10.1371/journal.pgen.1003691. 
666 Zhang, J., T. Zuo, D. Wang, and T. Peterson, 2014 Transposition-mediated DNA re-replication

667 in maize. eLife, Volume 3. https://doi.org/10.7554/eLife.03724.001.

668

669 Zhang, P., S. Chopra, and T. Peterson, 2000 A Segmental Gene Duplication Generated

670 Differentially Expressed myb-Homologous Genes in Maize. The Plant Cell, 12(12), pp. 2311-

671 2322. https://doi.org/10.1105/tpc.12.12.2311.

672

673 Zhang, P., Y. Wang, J. Zhang, S. Maddock, M. Snook et al., 2003 A maize QTL for silk maysin

674 levels contains duplicated Myb-homologous genes which jointly regulate flavone biosynthesis.

675 Plant Molecular Biology, 52(1), pp. 1-15. doi: 10.1023/a:1023942819106.

676

677 Zhang, Z., S. Schwartz, L. Wagner, and W. Miller, 2000 A greedy algorithm for aligning DNA

678 sequences. Journal of Computational Biology, Volume 7, pp. 203-214. doi:

$679 \quad 10.1089 / 10665270050081478$. 


\title{
Transposon-induced inversions activate gene expression in Maize pericarp
}

\author{
Sharu Paul Sharma*, Tao Zuo* and Thomas Peterson ${ }^{* \dagger}$
}

\section{Supplemental Information}
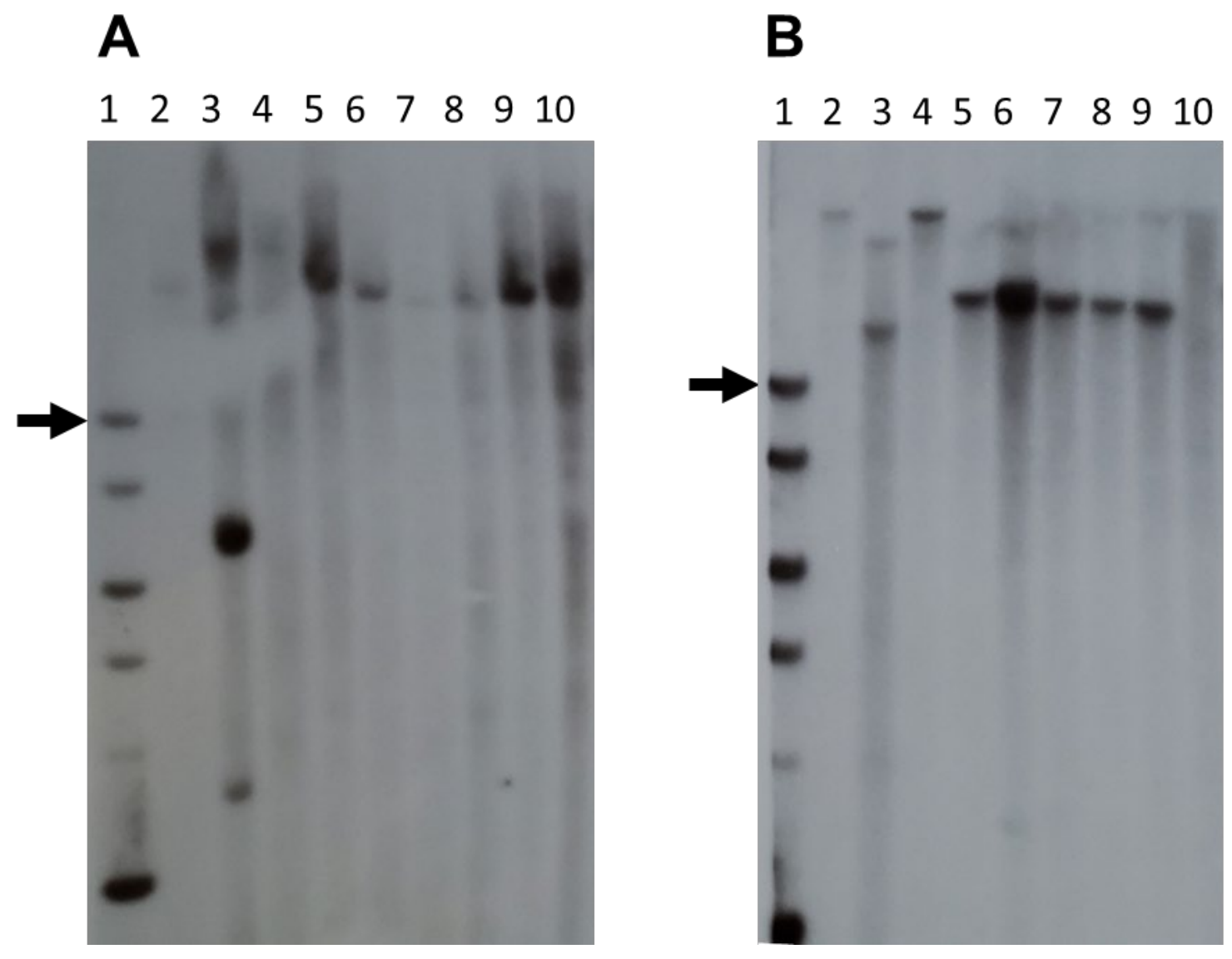

Figure S1: Southern Blot gel images using A) Hpal and B) EcoRV restriction enzymes with fragment-15 (within the $p 1$ enhancer) as a probe. Lane 1, DNA ladder, black arrow points to $10 \mathrm{~kb}$ fragment on each gel; Lane 2, J (p1-ww); Lane 3, P1-rr4B2; Lane 4, p1-wwB54; Lane 5, 140; Lane 6, E1; Lane 7, TZ3-4; Lane 8, SP1-18; Lane 9, S25; and Lane 10, TZ2-7. 
Figure S2: RT-PCR sequence aligned to $p 1$ and $p 2$ exons 1,2 and 3 . The middle sequence is RTPCR product from E1 (one of the inversions), the upper sequence is from $p 1$ and the lower sequence is from $p 2$. At three sites, SNPs in the RT-PCR product match $p 2$ (lower) but not $p 1$ (upper). Two additional SNPs in the RT-PCR product likely represent amplification or sequencing artefacts.

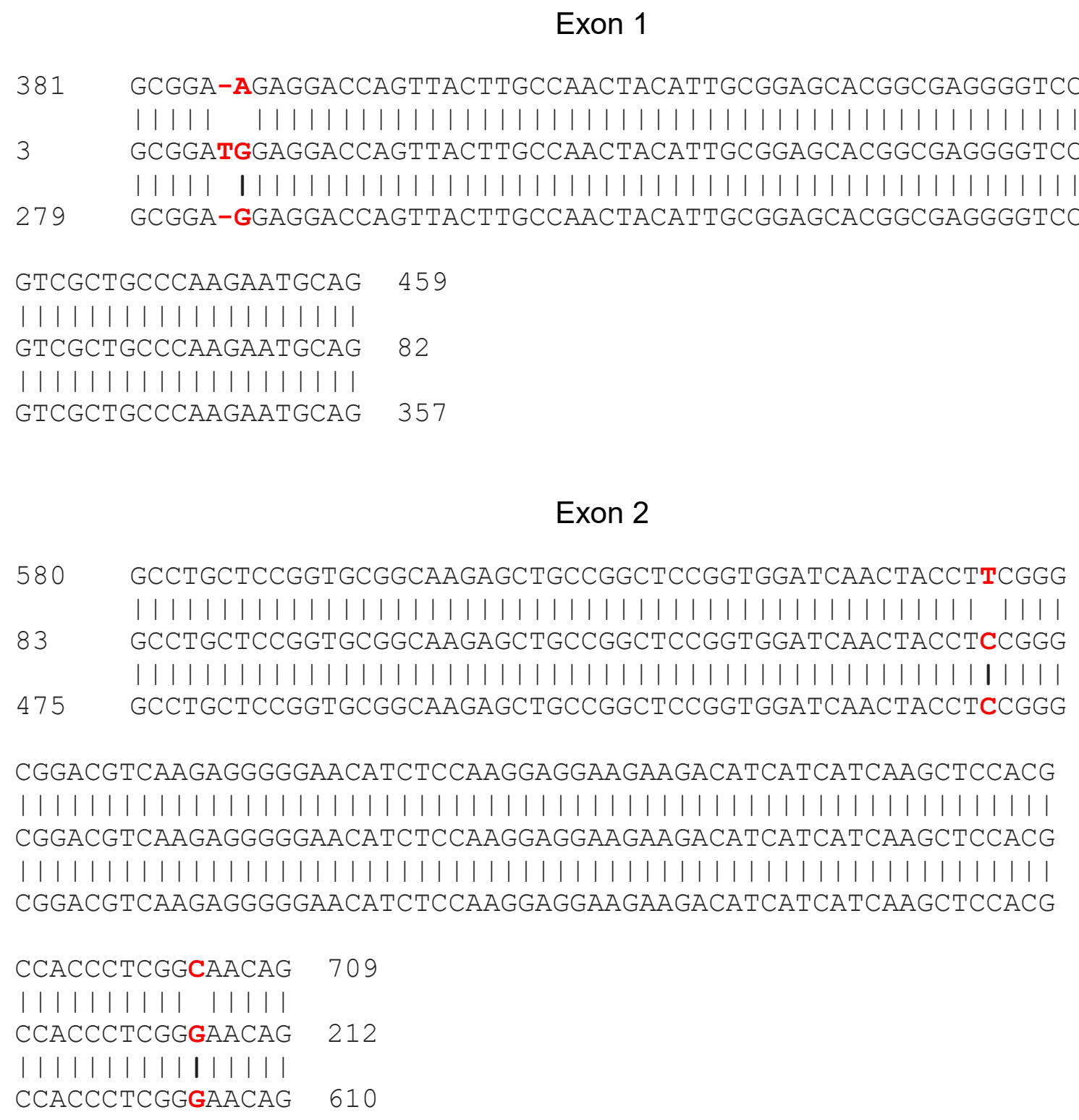

\section{Exon 3}

$\begin{array}{llll}5309 & \text { GTGGTCCCTGATCGCCAGCCACCTCCCC-GGCCGAAC } & 5344 \\ & |||||||||||||||||||||||||||||||||| \mid & \\ 213 & \text { GTGGTCCCTGATCGCCAGCCACCTCCCCCGGCCGAAC } & 249 \\ & |||||||||||||||||||||||||||||||||| & \\ 4425 & \text { GTGGTCCCTGATCGCCAGCCACCTCCCC-GGCCGAAC } & 4460\end{array}$


Table S1: Primers used for screening for inversions

\begin{tabular}{|l|l|l|}
\hline Set 1 & GAACAGTGATGGGAATGTTG & CTGCTAGCTGCTAGCTGTTAGGCTC \\
\hline Set 2 & GAGTCGCGAGCAGTGGAG & CTGCTAGCTGCTAGCTGTTAGGCTC \\
\hline$f$ Ac- $p 1$ junction & GACCGTGACCTGTCCGCTC & TGCCATCTTCCACTCCTCGGCTTTAG \\
\hline Nested & $\begin{array}{l}\text { GGCATAGTGAGACCCATTCCTC } \\
\text { CTTC }\end{array}$ & CCTCTCCATGAGCAATGTGTCTTAT \\
\hline
\end{tabular}

Table S2: Primers for sequencing inversion endpoints

Primers for $A c$ side

\begin{tabular}{|l|l|}
\hline \multicolumn{2}{|l|}{ Ac primer: GCTCTACCGTTTCCGTTTCCGTTTACCG } \\
\hline 140, E1, TZ3-4, SP1-18 & TTATACTTGCGACGCTGTGG \\
\hline S25 & TCTTTTGGCCATACGTCTCC \\
\hline TZ2-7 & CTTGGAGGACGAGGGATGGCAATGGG \\
\hline
\end{tabular}

Primers for $f A c$ side

\begin{tabular}{|l|l|}
\hline \multicolumn{2}{|l|}{$f$ A primer: CTGCTAGCTGCTAGCTGTTAGGCTC } \\
\hline 140, E1, TZ3-4, SP1-18 & GCAGCCTTTTCTTGCAGTCA \\
\hline S25 & CCCTCGTCCTCCAAGATTCTCCCCCTG \\
\hline TZ2-7 & GATTGGCTGAACCGTGACGT \\
\hline
\end{tabular}

Table S3: Primers used for RT-PCR

\begin{tabular}{|l|l|l|}
\hline$p 2$ & GCGGAGGAGGACCAGTTAC & CTGAGGTGCGAGTTCCAGTAG \\
\hline Beta-tubulin & CTACCTCACGGCATCTGCTATGT & GTCACACACACTCGACTTCACG \\
\hline
\end{tabular}


Table S4: Inversion alleles, Target Site Duplications, and relevant distances (in basepairs)

\begin{tabular}{|l|l|l|l|l|}
\hline Name & Target Site Duplication & $\begin{array}{l}\text { Distance } \\
\text { from TSS }\end{array}$ & $\begin{array}{l}\text { E-P } \\
\text { distance }\end{array}$ & $\begin{array}{l}\text { Size of Inverted } \\
\text { fragment }\end{array}$ \\
\hline 140 & CCGGTGGC & 3505 & 8235 & 81800 \\
\hline E1 & CAGCCAGG & 3387 & 8117 & 81682 \\
\hline TZ3-4 & TGTGTAGT & 3376 & 8106 & 81671 \\
\hline SP1-18 & GTCGGGGC & 3203 & 7933 & 81498 \\
\hline S25 & CTCGTCGA & 3070 & 7800 & 81365 \\
\hline TZ2-7 & ATCTCTTC & 2692 & 7422 & 80987 \\
\hline
\end{tabular}

